\title{
Chronic Ingestion of High-Fat Diet and Energy Restriction Modulates Expression of VEGF and VEGFR2 in Aged Rat Myocardium
}

\author{
D. Fernandes, I. Tomada, H. Almeida and D. Neves
}

Department of Experimental Biology, Faculty of Medicine and IBMC of Universidade do Porto, 4200-319 Porto - Portugal

Cardiovascular diseases (CVD) constitute a leading cause of death in the aged population. Regular intake of hyperlipidic diet and obesity can induce endothelial dysfunction that precedes the onset of atherosclerosis and CVD. High-fat diet regular consumption leads to structural modifications in heart tissue [1], and changes in the expression of angiogenic factors and their specific receptors [2]. Hence, we aimed to characterize the expression pattern of vascular endothelial growth factor (VEGF) and its membrane receptor VEGFR 2 in young and aged rat hearts under high-fat diet and energy restriction.

Adult male Sprague-Dawley rats (8-wks old), individually housed in appropriated cages with free access to tap water and maintained under a $12 \mathrm{~h}$ light-dark cycle at standard temperature $\left(20-22^{\circ} \mathrm{C}\right)$ and humidity (40-60\%), were divided into three groups $(\mathrm{n}=12)$ : a control group of rats fed ad libitum with a standard rodent chow with $4 \%$ of energy supplied from fat (C); a group of rats with free access to a rodent purified diet with $45 \%$ of energy supplied from fat (HF) and another subjected to energy restriction (75\% of daily individual intake of $\mathrm{C}$ rats) (ER). In each group, 6 rats were sacrificed by decapitation when they reached the ages of 6 and 18 months. The heart was removed and divided in two fragments that were immediately fixed in $10 \%$ buffered formalin, or frozen at $-80^{\circ} \mathrm{C}$ until analysis. Dual-labelling immunofluorescence detection of the endothelial protein PECAM (platelet/endothelial cell adhesion molecule) and $\alpha$-actin, VEGF and VEGFR2 was observed in an Apotome fluorescence microscope (Carl Zeiss MicroImaging $\mathrm{GmbH}$ ). Further, we performed morphometrical analysis of smooth muscle content in the myocardium after imunohistochemical detection of $\alpha$-actin employing the program Image ${ }^{\circledR}$ (National Institutes of Health, Maryland, USA). Semiquantification of VEGF and VEGFR2 was made by Western blotting followed by pixel computerized quantification (Scion Image $\left.^{\circledR}\right)$.

The expression of PECAM was restricted to the endothelium and $\alpha$-actin to smooth muscle layer of myocardium's blood vessels in all experimental groups. VEGF and VEGFR2 were detected in endothelium and in lower extension in the cardiomyocytes. It was verified that VEGF expression was significantly reduced in 18-months rats under consumption of high-fat diet when compared with agematched group, and that VEGFR2 expression significantly decreased during aging. Morphometrical study concerning the perivascular smooth muscle layer demonstrated a significant decrease in the ER groups $(0.80 \pm 0.07$ and $0.78 \pm 0.05$, for 6 and 18 months, respectively) when compared with agematched controls ( $1.16 \pm 0.07$ and $1.10 \pm 0.06$, for 6 and 18 months, respectively).

Overall, the data suggest that aging and fat content of diet modulate vascular mechanisms of the heart, nevertheless further molecular studies will be necessary to elucidate this hypothesis.

References

1. Siri-Tarino et al., Current Atherosclerosis Reports 12: 384-390, 2010

2. Ferrara et al., Nature Medicine 9: 669-676, 2003 

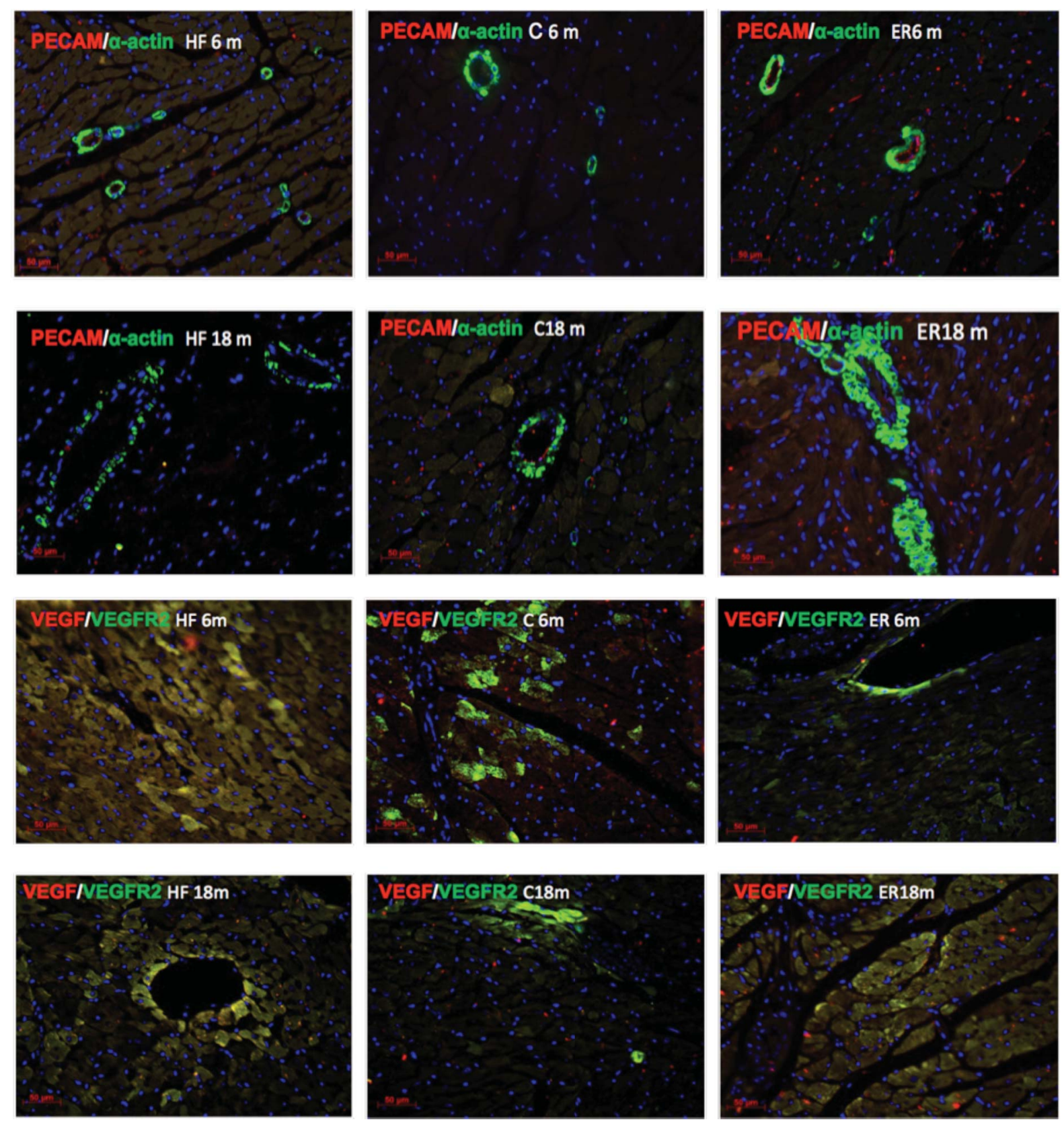

Figure 1. Dual immunolabelling of PECAM (red) / $\alpha$-actin (green) and VEGF (red) / VEGFR2 (green) in the heart of rats from all experimental groups. Note co-localization of VEGF and VEGFR2 in the endothelium (yellow). 